\title{
Development of a ground displacement measurement method for failed slopes at the toes of landslides
}

\author{
K. Higuchi ${ }^{1}$, K. Fujisawa ${ }^{1}$, M. Tohei ${ }^{1}$, S. Okawa ${ }^{2}$, H. Shimomura ${ }^{3}$, and T. Sakata ${ }^{4}$ \\ ${ }^{1}$ Public Works Research Institute, Japan \\ ${ }^{2}$ KOWA Co., Ltd., Japan \\ ${ }^{3}$ PASCO Co., Ltd., Japan \\ ${ }^{4}$ RADIC Co., Ltd., Japan
}

Received: 18 June 2007 - Revised: 19 September 2007 - Accepted: 19 September 2007 - Published: 2 January 2008

\begin{abstract}
Sediments produced by a slope failure of the toe of a landslide are assumed to prevent the spread of the failure. Thus, sediment removal is an emergency measure that must be done safely while the slope is observed. Observation must also be done safely without requiring workers to enter the failed slope to avoid secondary disasters.

Generally, a non-prism type totalstation is useful to measure ground displacement under such circumstances, because this type of totalstation can be used without placing targets on the slope. However, the lack of prisms results in problems related to the objectivity of measurement data, collimation error of the totalstation, measurement at night, and so on.

The authors have developed targets and a remote target placing method in order to resolve the above problems. As a result, we have established remote measurement technique for ground displacement on failed slopes at the toes of landslides. This paper describes the measurement technique that has been developed.
\end{abstract}

\section{Introduction}

Experience confirms that small scale landslides and failed slopes at the toe and on the boundaries of landslide blocks cause disasters. There is a strong possibility that sediments consisting of displaced soil at the toe or boundaries of a landslide may prevent the expansion of the landslide soil mass behind it or of the failure itself. But there are cases where failed soil must be removed, even temporarily, as an emergency measure to protect human life or to ensure that a road is open to traffic. Removal work is executed carefully by, for example, posting observation staff to ensure safety, but there is a danger that the work will induce a landslide behind the site or cause a repeat failure, resulting in a secondary disas-

Correspondence to: K. Higuchi

(higuti44@pwri.go.jp) ter. And to study countermeasures, it is essential to carry out an emergency survey of landslide, but this survey must be performed safely.

The work can be done while objectively evaluating its safety if it is possible to remotely measure changes on a slope that is at risk of failure in this way. Methods of measuring the quantity of change from a remote location are image analysis and laser scanners. But because these methods do not use targets (prisms etc.), their measurement precision is about $10 \mathrm{~cm}$ (Asano et al., 2001) and it is difficult to state that they provide precision adequate to grasp the change of slopes that are the precursors of a repeat failure.

On the other hand, a non-prism type totalstation is a method of measuring displacements in millimeter units from a remote location without the use of targets. At the site of a slope failure that occurred on the left bank of the Sanashi River temporarily blocking the river course in Shimooritate, Niigata Prefecture (Fig. 1, Sabo Technical Center, 2004), this method was used for measurements at an observation distance of $130 \mathrm{~m}$, confirming a maximum error of $9 \mathrm{~mm}$ that exceeded the precision of the instrument $(3 \mathrm{~mm})$. This error occurred at times of stable measurement values free of the impacts of mist, accumulated snow, etc., and the possibility of observation error caused by the inability to measure a single location accurately was high. Additionally at night, there were cases where it was impossible to perform measurements, because of low visibility (Yuzawa Sabo Office, 2004). And at failure slopes at the toes of landslides, the distance from the slope to places where observations can be made safely is longer than in a case where measurements are performed to control work safety etc., so there are conditions in which such collimation errors occur easily. Therefore, even when a non-prism type totalstation is used, a target of some kind is necessary, and in emergencies, measurement technology that can be used to observe slopes at night is necessary.

Published by Copernicus Publications on behalf of the European Geosciences Union. 


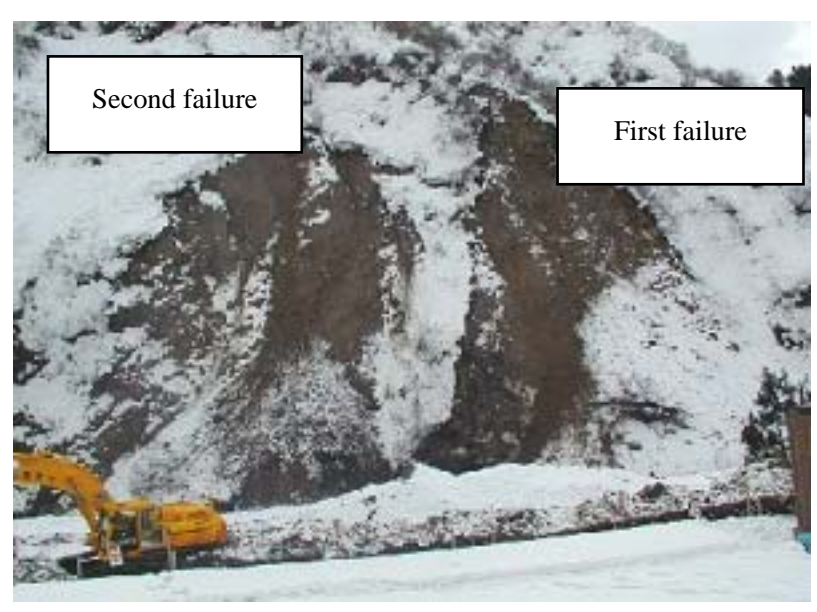

Fig. 1. Slope failure on left bank of the Sanashi river.

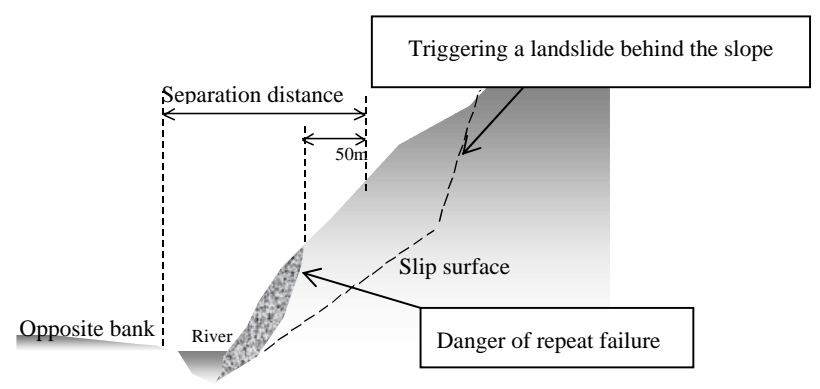

Fig. 2. Slope failure at toe of landslide.

So the authors have developed a method of measuring displacement from a remote location by placing targets on a failed slope without having any workers walk on the dangerous slope. This report describes this newly developed measurement method.

\section{Characteristics of failed slopes and existing measure- ment technologies}

\subsection{Characteristics of failed slopes}

As shown in Fig. 2, a failure at the foot of a slope may be the toe of a landslide above it, and the failed sediments must be removed carefully, because of the danger of a secondary disaster.

Using documents concerning landslide disasters from the Public Works Research Institute's Landslide Research Team (1999) and the Sabo Technical Center (2001), 23 cases of such failure of the toe of a landslide were selected from among a total of 376 such cases. These all occurred facing rivers or the ocean. Of these, 6 faced the ocean, and the other 17, faced rivers as shown in Fig. 2. An examination of the soil layers of these slopes has revealed that $70 \%$ are rock or

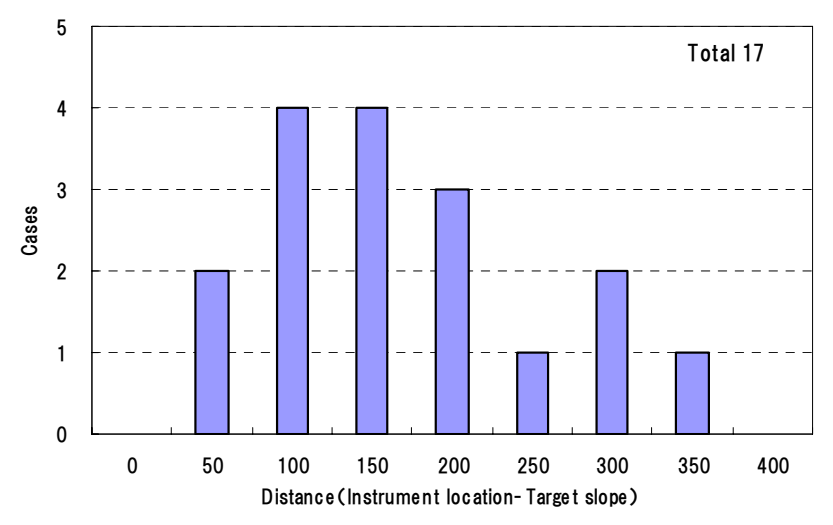

Fig. 3. Distance from instrument location to slope necessary for safe measurements.

colluvium and $30 \%$ are clayey soils that they are of diverse kinds with no geological trends observed, and they have igneous rocks, sedimentary rocks, metamaorphic rocks and so on. According to the above 17 cases facing river, the required distances to measure the slope safety was approximately 50 to $350 \mathrm{~m}$ as shown in Fig. 3 .

\subsection{Existing measuring technologies}

\subsubsection{Visual observations}

Visual observation by a person is an extremely effective method, because it is a method adaptable to circumstances and it permits comprehensive judgments. But on the other hand, its usefulness is limited by the decrease in observers' concentration over time, their inability to detect slow change, and a lack of objectivity retarding the location observed and the measured data. And it is dangerous for an observer to enter the failed slope area.

\subsubsection{Ground extensometer and optical fiber sensor}

Ground extensometer's have been widely used for emergency measurements when slope disasters have occurred, but there are problems with their use: the need for workers to walk on a dangerous slope to install them and the time until measurements begin. And because their measurement range is limited to between 20 and $40 \mathrm{~cm}$ (Japan Association for Slope Disaster Management, 1997), when displacement exceeding their measurement range has occurred, a worker must reenter the slope to reset the devices. In recent years, measurement methods using optical fiber have been used, but they suffer from the same shortcomings as ground extensometers.

\subsubsection{Totalstation (prism type)}

This widely used remote slope observation method can be used to perform high precision measurements from long dis- 
tances. If it is a type equipped with an automatic tracking system, it permits automatic unmanned observations and can measure each location in a few seconds (Association of Precise Survey and Applied Technology, 1995). But, when it is used for observations using a prism type that applies a light beam, a prism or reflective sheet must be placed on the target slope, and these must be installed without requiring a worker to walk on the dangerous slope.

\subsubsection{Totalstation (non-prism type)}

Totalstations include the non-prism type that applies a laser beam and does not require a prism or reflective sheets. Although inferior to the light beam type in 2.2.3, it can perform high precision long-distance measurements. And because it cannot perform automatic tracking observations, it is used manually, but its measurement time per location is the same as when using the prism type (Association of Precise Survey and Applied Technology, 1995).

It is, therefore, a useful method of measuring dangerous slopes off-limits to workers. But, the reflectivity of the object of the observation influences its precision, so measurement is impossible if the reflectivity is low. When multiple observation points are measured repeatedly, the characteristics of the observation points depend on the memory of the observers, so objective evaluations of whether a single point can be measured are not ensured.

\subsubsection{Three-dimensional laser scanners, image analysis,} GPS

In addition to totalstations, remote observations can be made using 3D laser scanners, image analysis, GPS, etc. A 3-D laser scanner can make plane observations, permitting the quantity of movement to be clarified in three-dimensions. But, it takes several hours to complete the analysis process, although it can perform a measurement in a few tens of seconds (Association of Precise Survey and Applied Technology, 1995). Similarly to photo image analysis, processing time is necessary to obtain the stipulated precision. When GPS is used, workers must walk on the slope to install the instruments as in the case of ground extensometers. And calculation such as time averaging is needed to obtain the stipulated precision, so although it is an effective method of performing long-term observations, it is difficult to apply it to emergency sites where judgments are needed in real time.

\section{Newly developed measurement technology}

3.1 Selecting conditions and measuring instruments necessary to measure a failed slope

The following conditions 1 to 3 must be considered in order to safely and precisely measure the ground displacement on a failed slope at the toe of a landslide.
Table 1. Spot diameter of the optical beam and the laser beam.

\begin{tabular}{ccc}
\hline $\begin{array}{c}\text { Measurement distance } \\
(\mathrm{m})\end{array}$ & $\begin{array}{c}\text { Optical beam } \\
(\mathrm{mm} \times \mathrm{mm})\end{array}$ & $\begin{array}{c}\text { Laser beam } \\
(\mathrm{mm} \times \mathrm{mm})\end{array}$ \\
\hline 10 & $15 \times 26$ & $4 \times 7$ \\
20 & $29 \times 58$ & $7 \times 14$ \\
50 & $73 \times 145$ & $10 \times 20$ \\
100 & $145 \times 291$ & $12 \times 40$ \\
200 & $291 \times 582$ & $25 \times 80$ \\
300 & $437 \times 873$ & $38 \times 120$ \\
500 & $727 \times 1,454$ & $60 \times 200$ \\
\hline
\end{tabular}

1. Remote placing and measurement can be done. Because failure locations are often steep, and are at danger of a repeat failure or other secondary disaster, such sites are often off-limits to workers. Therefore, it must be possible to install the equipment and use it to perform measurements from a location remote from the failure site.

2. Measurement can be done quickly in an emergency. It is often impossible to predict where and when a landslide or slope failure will occur. After a disaster has occurred, response measures must be taken as quickly as possible to save lives and prevent the spread of the disaster. The observation equipment, should, therefore not be special equipment difficult to obtain, but equipment available on the market so it can be acquired quickly.

3. It can detect change of a few millimeters. On a failed slope, a slight change may be a precursor of a repeat failure. It is, therefore, necessary to accurately detect ground movement of a few millimeters.

As a result of a study of equipment that satisfies the above conditions, the measurement device suitable for measurement of failed slopes at the toe of a landslide that was used was the Geographical Survey Institute registered totalstation Grade 2 Class A (distance measurement precision $2 \mathrm{~mm} \pm 2 \mathrm{ppm}$ ), Japan. Of course, if it is possible to obtain a more precise type during an actual disaster, a higher class totalstation than Grade 2 Class A is used. Because the longer the observation distance, the greater the error caused by angle measurement, so only the measurement distance was used. And as stated in 2.2, even when based on a light beam or when based on a laser beam, a reflective object or other target is necessary to improve measurement performance. 


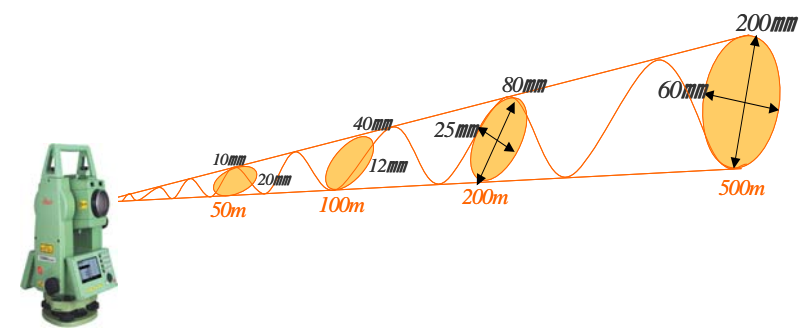

Fig. 4. Image of the spot diameter of the laser beam 9.

\subsection{Development of the target and placing method}

\subsubsection{Development of the target}

\section{Target conditions}

Regarding the spot diameters of the light beam and of the laser beam of an totalstation, as shown in Fig. 4 (Ruege, 1990), as the distance increases, the elliptical spot expands as it becomes distorted. As the elliptical spot expands, the lengths of the short axis and long axis of the ellipse vary according to the distance as shown in Table 1, and the spot diameter of a light beam is larger than that of the laser spot at the same distance.

As shown by Fig. 3, it is necessary to measure at distances between 50 and $350 \mathrm{~m}$, but for the target (reflective mirror) used at about $300 \mathrm{~m}$, it has been standardized at a diameter of $63.5 \mathrm{~mm}$ with a single prism, and at $60 \times 60 \mathrm{~mm}$ with a reflective sheet. Therefore, if a target with approximately the same capacity of the standardized target is used, the diameter may be about $65 \mathrm{~mm}$, but if its capacity is not the same, it is necessary to use a target with diameter larger than the spot in order to obtain the reflectivity necessary for measurements.

\section{Development of the target}

A glass capsule (length: $79 \mathrm{~mm}$, diameter: $10 \mathrm{~mm}$ ) containing paint like that shown in Fig. 5 was developed considering the above conditions. The paint contained glass beads with diameter of $50 \mu \mathrm{m}$ to improve its reflectivity. Regarding the color of the paint, it is generally said that the reflectivity of white is high, but the results of laboratory investigations of the reflectivity by 11 colors of light beams and of laser beams of totalstations revealed that the highest reflectivity was that of pink as shown in Fig. 6. So pink paint was selected. One milliliter of paint that can form a target with diameter of $65 \mathrm{~mm}$ was used. Causing this capsule to strike the observed slope to form a target creates a collimation mark and improves reflectivity. And even in cases where it is impossible to see a target that has been placed on a slope, the location of the target can be specified by confirming the reflectivity of light beam or laser beam.

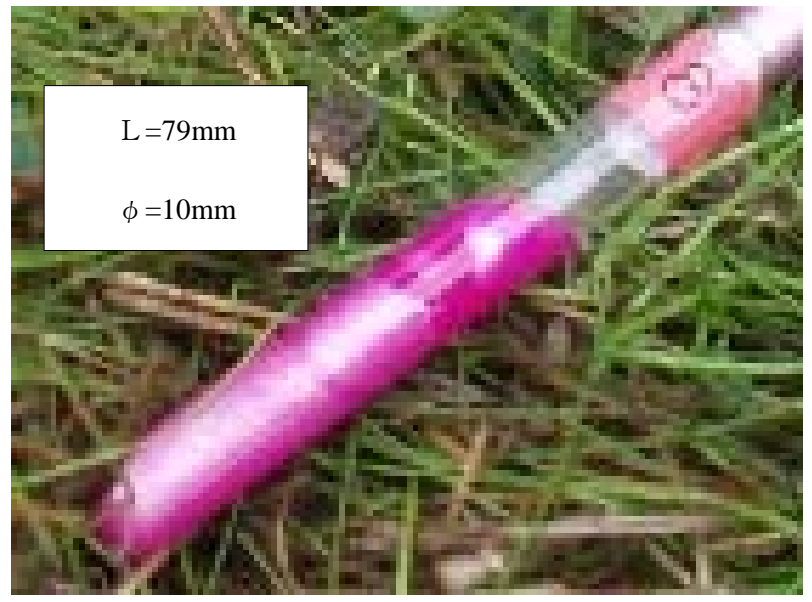

Fig. 5. Glass capsule containing the paint.

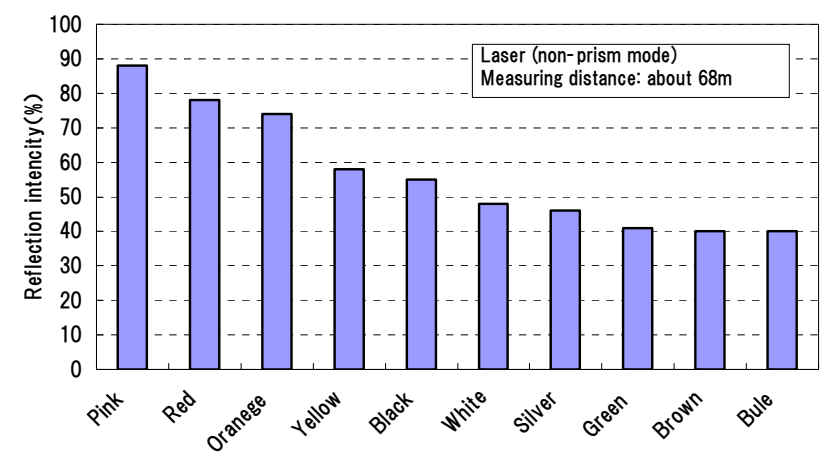

Fig. 6. Paint color and reflectivity.

\subsubsection{Development of placing method}

\section{Placing conditions}

As shown by Fig. 3, the distance from the slope needed to measure it safely ranges from 50 to $350 \mathrm{~m}$, so the placing of targets must be done from an equally distant location. And they must be placed accurately at specified locations such as unstable rock mass. And because it is assumed this method will be applied in emergencies, it should be a method that can be implemented by anyone in an emergency rather than a method requiring staff with special skills, qualifications, or certification: the use of a radio controlled helicopter or methods of throwing the targets using explosives for example.

\section{Development of the placing method}

A method using a crossbow (Bow Gun) of the kind shown in Fig. 7 was developed considering the above conditions. A crossbow may be used by anyone who is 18 years of age or older without the need for qualifications or certification, and 


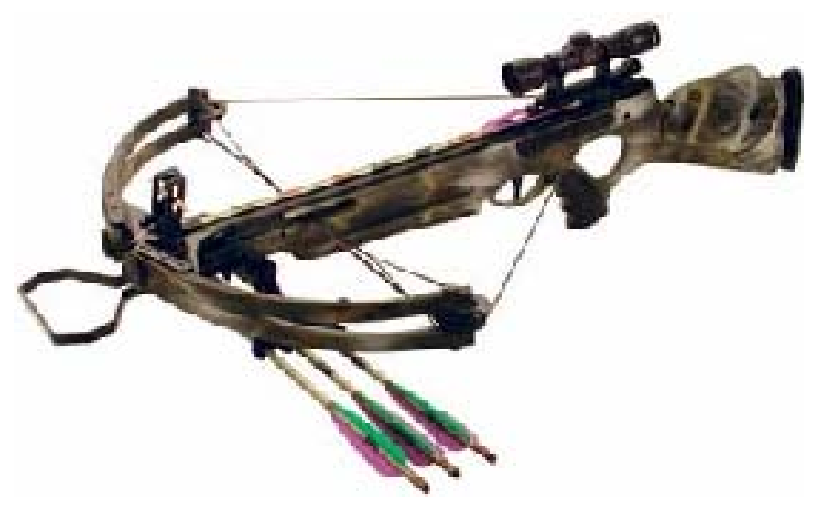

Fig. 7. Crossbow (Bow Gun).

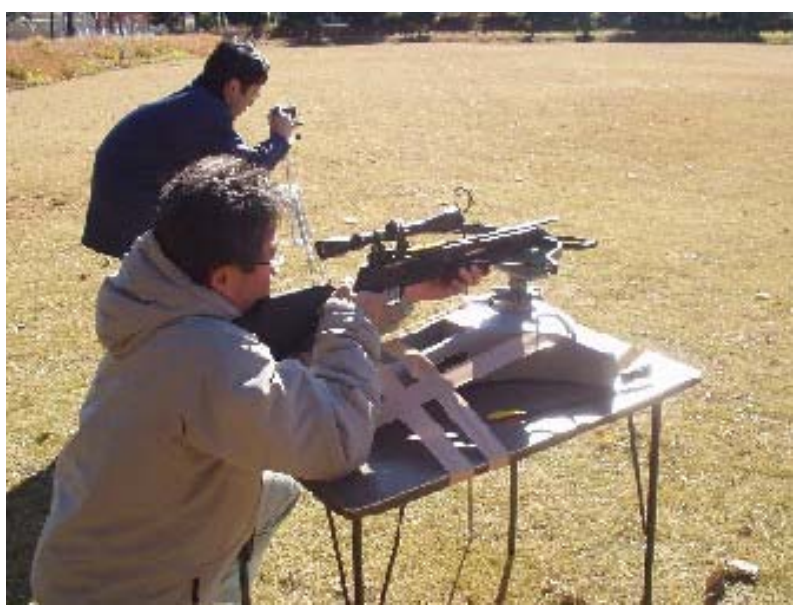

Fig. 8. Trial firing of the crossbow.

without restrictions under air transportation regulations. As shown by Figs. 8 and 9, a person using it for the first time can strike the target with stipulated precision without any special skill. Mounting the paint-filled capsules described above on the tips of the arrows of the crossbow as shown by Fig. 10, then shooting the arrows can form a target on a slope $300 \mathrm{~m}$ from the crossbow with a positional precision of $\pm 30 \mathrm{~cm}$. Of course shortening the distance improves the precision. Targets can be placed using a crossbow, but to improve its accuracy, a shooting base that can set the horizontal angle and the angle of elevation as shown in Fig. 11 was developed. This permits the targets to be placed at the intended locations easily no matter who is shooting them. The remote monitoring method shown in Figs. 12 and 13 was developed by developing the above targets and placing method.

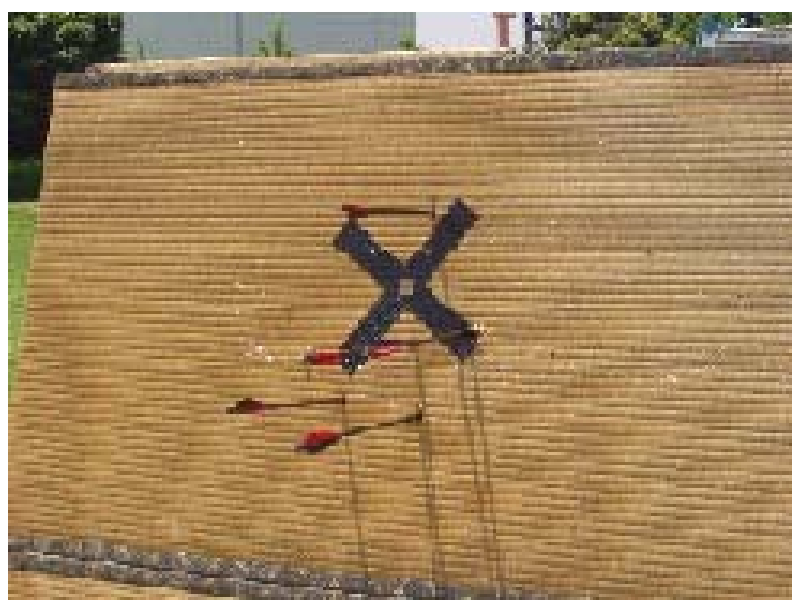

Fig. 9. Mark $100 \mathrm{~m}$ from the firing point.

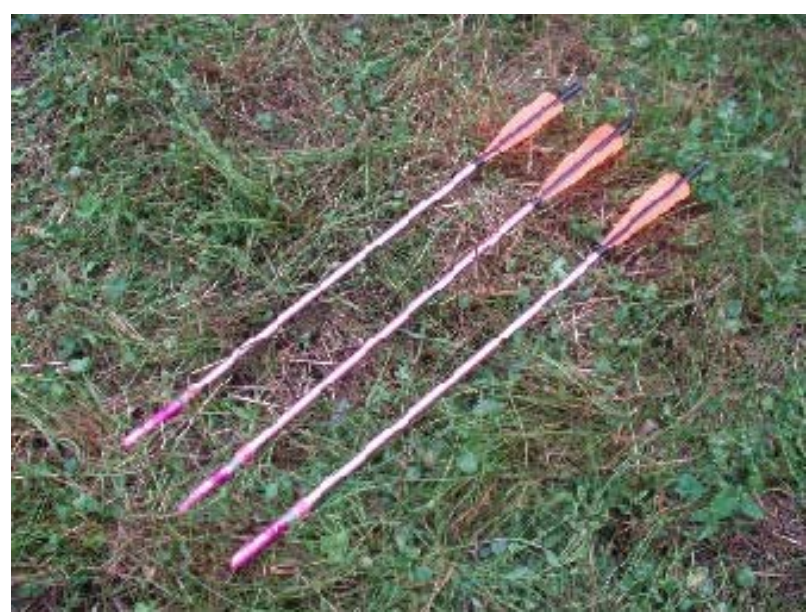

Fig. 10. Arrows equipped with paint-filled capsules.

\section{Confirmation of performance by a field test}

In order to confirm the suitability of the targets and placing method that were developed, a test was performed on riprap downstream of a rock fill dam assuming it was a failed slope as shown in Fig. 14. Before the main test, a test was attempted on an actual failed slope, but because it was impossible to walk on the slope to confirm the state of placing of the targets, an almost full-size rock fill dam similar to an actual slope was used in place of an actual site.

The test was performed at the Surikamigawa Dam operated by the Tohoku Regional Development Bureau of the MLIT. The stones of the downstream riprap at the site are mainly granite and sandstone, and are the types of stone found at the site of actual slope failures. And the gradient of the riprap is gentle at $27^{\circ}$, so it was possible to safely visually confirm the way the targets were installed. 


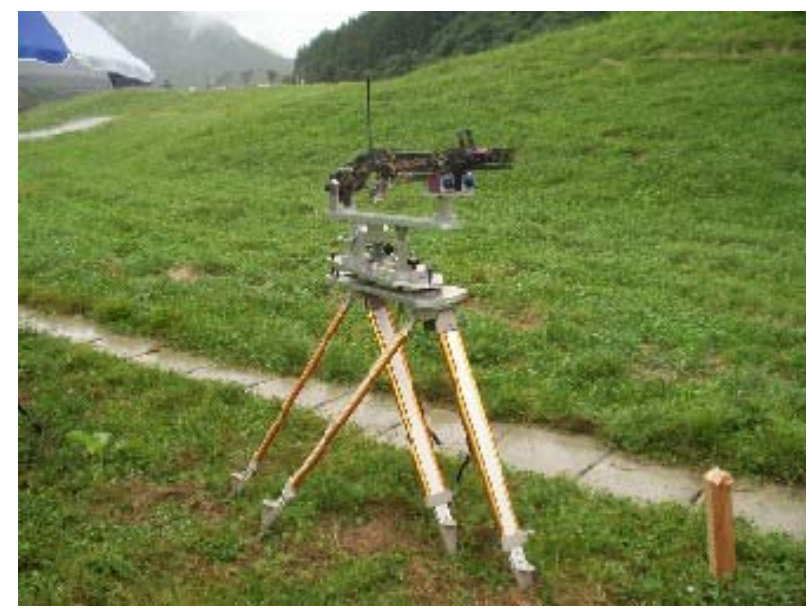

Fig. 11. Crossbow firing stand.

\subsection{Crossbow placing performance}

To confirm the capability of the crossbow firing stand to control the arrows, arrows were shot from locations ranging from 50 to $350 \mathrm{~m}$ downstream from the toe of the slope at angles of elevation ranging from 5 to 45 degrees, confirming the shooting accuracy and this was accompanied by a trial shooting test to set the shooting angle of elevation.

The arrows were shot in the same direction along a base line, so the arrival points are generally along the length of the base line, but those shot at a greater angle of elevation, or in other words, shot higher, were slightly influenced by the wind. But as stated above, because the shooting accuracy was originally $\pm 30 \mathrm{~cm} / 300 \mathrm{~m}$, as long as the wind was not strong enough to exceed $10 \mathrm{~m} / \mathrm{s}$, it is possible to place an arrow almost exactly at the desired location.

Figure 15 shows the arrival points of arrows viewed longitudinally. The plotting in the figure shows the arrival points on the riprap when the shooting point is horizontal distance of $0 \mathrm{~m}$ and its height is $0 \mathrm{~m}$, and the curves are the theoretical curves that represent the courses of the arrows. These show that arrows arrived at locations that generally conform to the theoretical curves, and it is possible to confirm that the targets can be placed at the intended locations by setting the angle of elevation of shooting. The theoretical curve was induced based on an equation of momentum that is calculated based on the shape of the paint-filled capsule and arrow, and by hypothesizing that the air resistance is proportional to the square of the speed.

It is predicted that the graph obtained in this way will be used as a simple table to set the shooting angle of elevation of the arrows as quickly as possible in an emergency. It has been confirmed that shooting can start about five minutes after a worker assembles the crossbow shooting base, and that measurement can be started quickly, even during an emer-

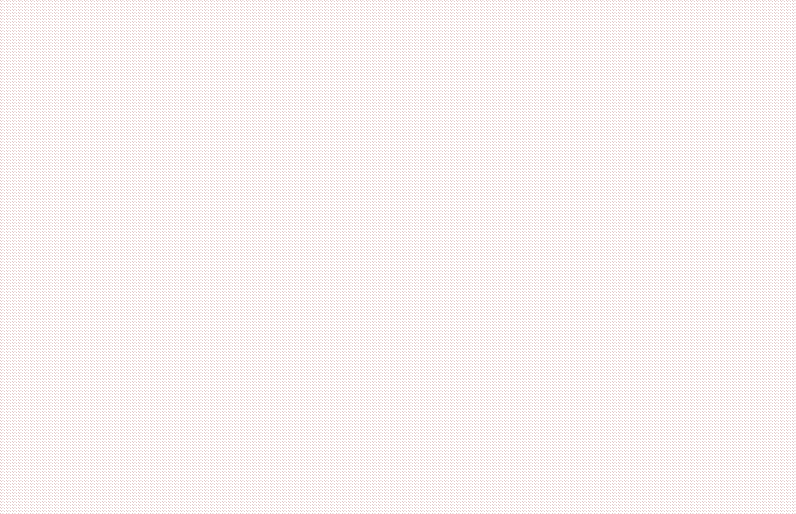

Fig. 12. Placing targets.

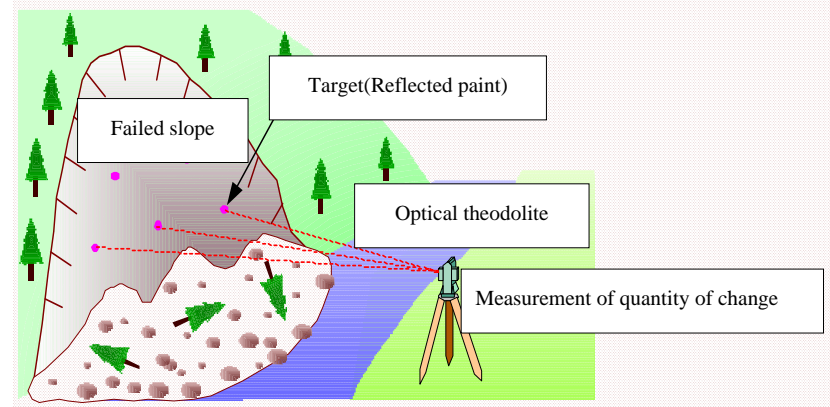

Fig. 13. Monitoring image.

gency, if the shooting location and observation location can be set.

\subsection{Target performance}

In order to confirm that a target shot by a crossbow has diameter of $65 \mathrm{~mm}$ or more on a slope and that the stipulated reflectivity is obtained by the totalstation, the targets formed were confirmed visually at the same time as their reflectivities were measured by the totalstation. And because actual slope disasters occur during rainy weather, the confirmation was done for a rainy weather case and for a clear weather case.

\subsubsection{Shape and reflectivity of the targets}

Figure 16 shows the shape of a target. As shown by Fig. 17, they are not only circular but also elliptical according to the angle that the arrow strikes the rock, but it confirms that targets with a diameter of about $65 \mathrm{~mm}$ will be formed at all locations.

Both the distance and the reflectivity of the targets were measured using both light beams (prism mode) and laser beam (non-prism mode). Results showed it was impossible to perform measurements using light beams because light 


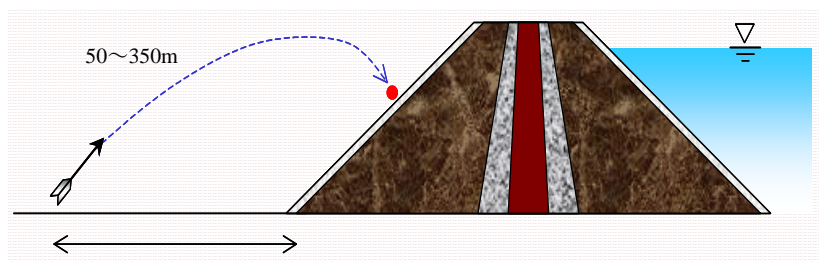

Fig. 14. Field test image.

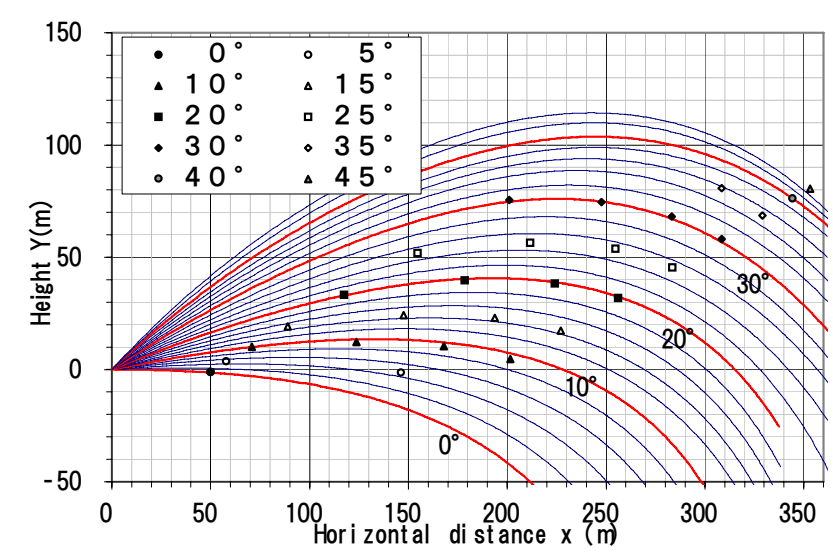

Fig. 15. Theoretical curves and actual data of arrow tracks.

beams could obtain only 0 to $1 \%$ of the necessary reflectivity (about $3 \%$ or more). In contrast, laser beams obtained reflectivity from $10 \%$ to $99 \%$ that is sufficient for measurement, even at an observation location where the reflectivity without a target is about $1 \%$. For such reasons, unless otherwise necessary, reflectivity as used below refers to the reflectivity of a laser beam.

Reflectivity of targets ranges from a few times to several tens of times that of the area surrounding the targets, and it was confirmed that it is distributed around the point where it is highest as shown in Fig. 18. This result has confirmed that even when a target is so far that it cannot be seen by the naked eye or using the binoculars on the totalstation, measurement can be done by repeatedly measuring a single location by confirming a point with strong reflectivity. Figure 19 shows the target reflectivity - observation distance (distance from the totalstation to the target) relationship. As the observation distance increases, the reflectivity falls abruptly, but at a horizontal observation distance of $250 \mathrm{~m}$ for example, there is reflectivity of approximately $10 \%$, and it is reflectivity ranging from a few times to several tens of times as that in a case without a target as described above. Reflectivity measured about 2 months after placement is either equal to or higher than it is immediately after placement, confirming that a target does not lose its function as time passes. At the points enclosed by the broken line, the reflectivity is low, because targets are formed in crevices between rocks and in the shadows of irregularities on the surface that cannot be

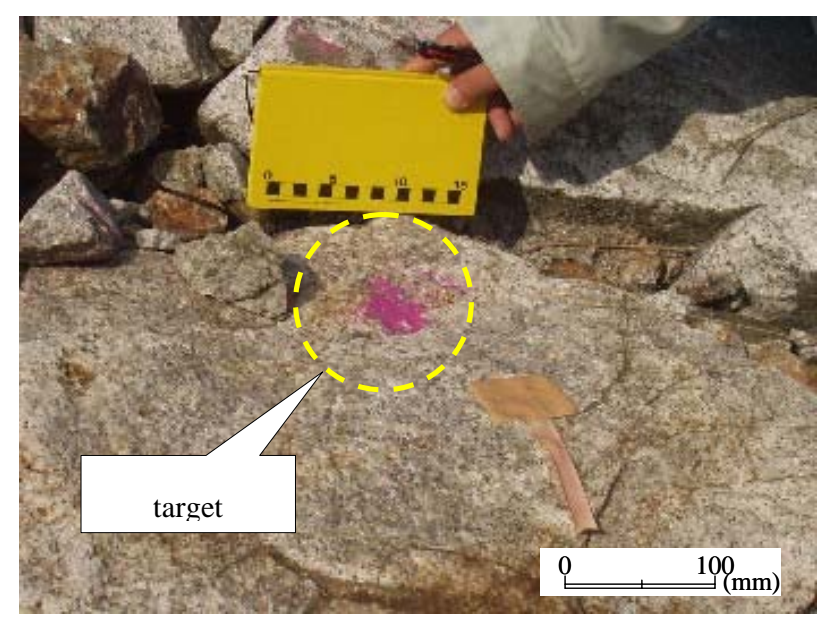

Fig. 16. Example of a target formed on the slope.

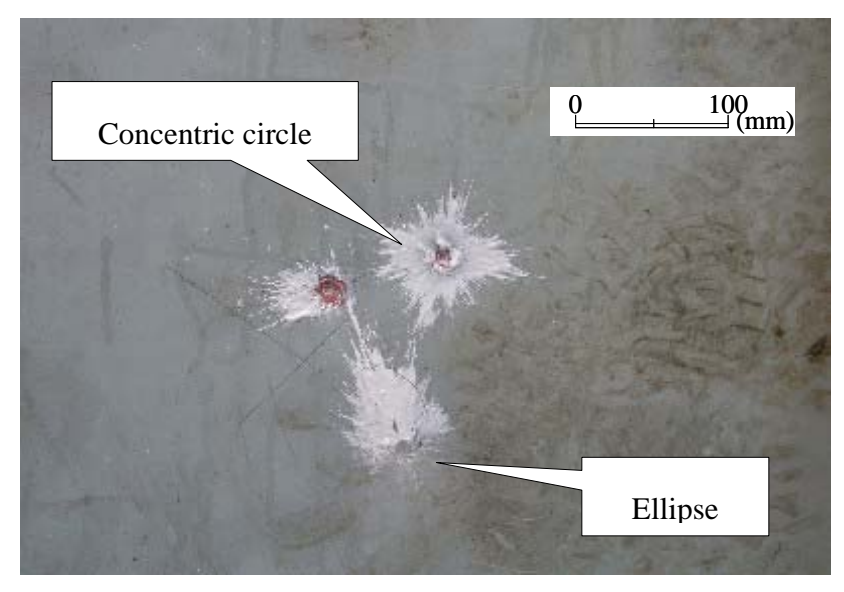

Fig. 17. Examples of target shapes.

reached by a laser beam as shown in Fig. 20. Presumably this will occur at actual sites, and in such cases, supplementary targets should be placed. If attention is focused only on whether or not measurement can be done using an totalstation, measurement is possible at a reflectivity of about $3 \%$, and when the newly developed target is used, it is possible to perform remote measurements over long distances. But targets are placed so it is possible to reduce collimation error by repeatedly accurately observing a single point, which means that it is necessary to treat the range up to $250 \mathrm{~m}$ where the difference between the reflectivity of the target and of its surroundings can be clearly distinguished as the range where measurements can be made with high precision.

\subsubsection{Nighttime measurement}

The reflectivity of the locations where the targets are placed are identical in the daytime and nighttime, confirming that the locations of the targets are accurately measured, even at 


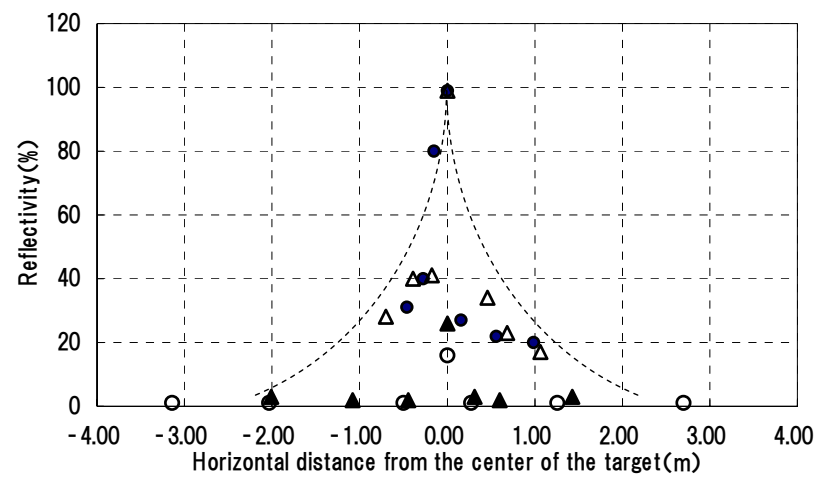

Fig. 18. Distribution of reflectivity near the target.

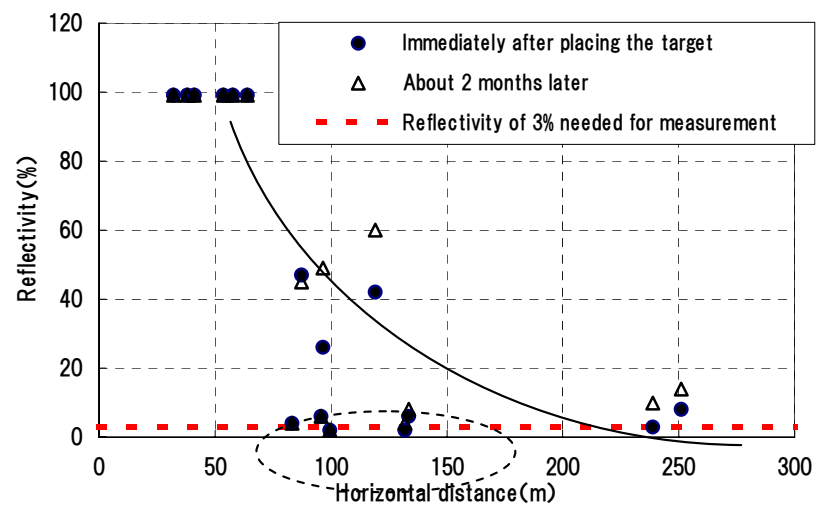

Fig. 19. Reflectivity - horizontal distance relationship.

night. The distribution of the reflectivity near the target is identical to that in Fig. 18. This has confirmed that every step from placing targets to measurements can be done at night by the same procedure as during the day.

During the daytime, the longer the distance, the more difficult it is to visually confirm the position of the targets, but at night on the other hand, there is no sunlight, so it was possible to visually confirm the locations of the targets not only with a laser beam, but with the optical beam of a flashlight. Therefore, it is assumed that the efficiency of work performed with nighttime measurements is not remarkably lower than that during the daytime.

\subsubsection{Impact of rainwater etc.}

If a slope has already been drenched by an earlier rainfall, even if the paint adheres to the rock, it may we washed off by rainfall. It was confirmed that even when a target has been formed, if a water membrane is formed by intense rainfall etc., the reflectivity falls sharply as shown in Fig. 21. But if the water membrane disappears as time passes, the reflectivity is restored.

The above results have confirmed that the paint-filled capsules that were developed adequately fill their functions as

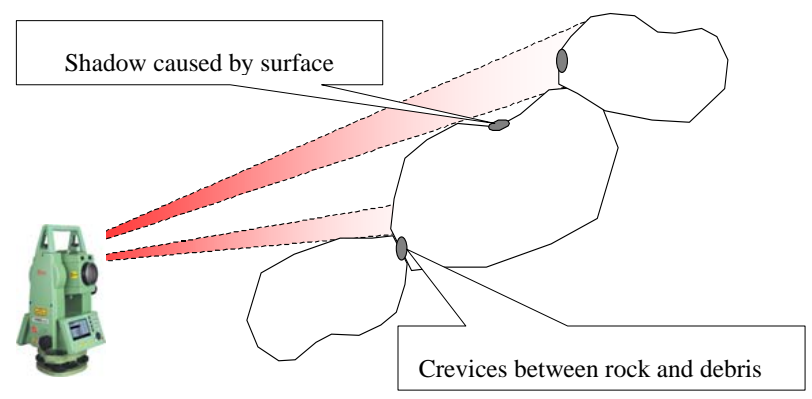

Fig. 20. State of locations where target reflectivity was extremely small.

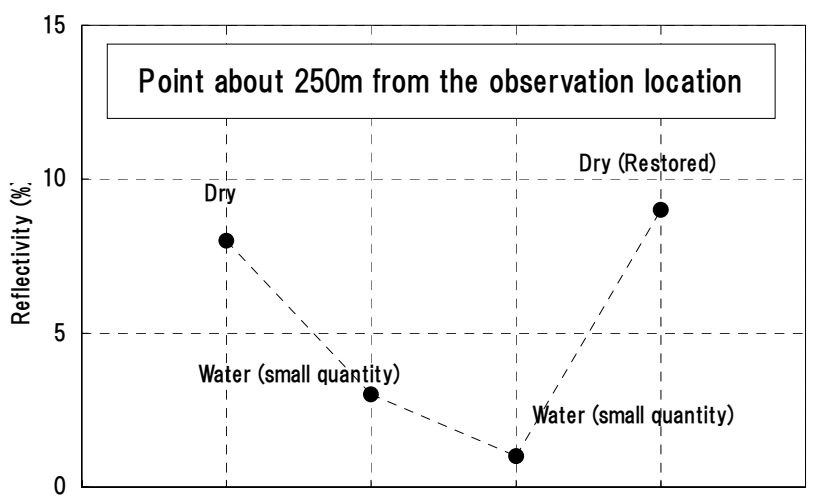

Fig. 21. Drop in the reflectivity caused by rainwater.

targets so they can be applied effectively, but the paint adhering to wet rocks etc. must be improved.

Incidentally, it was confirmed that the adhering paint on dry condition does not peel off even when sprayed by water under high pressure of $120 \mathrm{kPa}\left(\doteqdot 1.2 \mathrm{kfg} / \mathrm{cm}^{2}\right)$.

\section{Conclusions}

The authors have developed a method of remotely installing targets for an totalstation as a method that permits the measurement of ground displacement on a slope at risk of secondary disasters such as the failure of the slope at the toe of a landslide, from a remote location with good precision and safely without walking on the slope. Because this permits the use of a non-prism type of totalstation to eliminate collimation errors, which are assumed to have the greatest impact, it also permits measurement with precision that is extremely close to instrument precision that is specified in the instrument catalog. Below, the present achievements and future challenges of this method are separately summarized.

\subsection{Targets}

Capsules containing a mixture of paint and glass beads were developed to reduce collimation errors by clearly showing 
the location of measurement points and improving their reflectivity. This has improved the reliability of measurement data above that possible in the past using non-prism measurements. However, during rainfall, its performance as reflective target material declines. Therefore, the quality of the paint must be improved so that it will adhere to wet rock. Another future challenge is studying targets that can be used to perform measurements when the transported and deposited material is soil.

\subsection{Placing method}

A method of using a crossbow to place targets safely from a distance without requiring any workers to walk on the dangerous slope has been developed. The development of the placing method included the development of a shooting stand that controls the angle of elevation of the arrows, permitting the placement of targets with a positional precision of $\pm 30 \mathrm{~cm}$ from a distance of about $300 \mathrm{~m}$ by a worker without special training. In the future more data concerning angle of elevation and distance must be accumulated to improve the precision of the simple table used to set the angle of elevation. An application has been made for patents on the target and the placement technology that has been developed.

Acknowledgements. The Surikamigawa Dam Management Office of the Tohoku Regional Development Bureau of the Ministry of Land, Infrastructure and Transport generously provided the test site used for the field test. The authors wish to conclude this report by expressing their deep gratitude to everyone who cooperated with the performance of this study.

Edited by: P. Fabian

Reviewed by: two anonymous referees

\section{References}

Asano, H., Ishii, Y., and Tsunaki, R.: Study of Quantity of Landslide Movement Using a 3D Laser Scanner, Proc. of the Fortieth Conference of the Japan Landslide Society, 279-281, 2001.

Sabo Technical Center: Sanashi River Slope Failure Countermeasure Study Committee: Field Survey Document, 2004.

Yuzawa Sabo Office, Hokuriku Regional Development Bureau, Ministry of Land, Infrastructure and Transport: Feb. 10. Report on Emergency Restoration Countermeasure Work following the Sediment Failure Disaster on the Sanashi River in Yunotanimura, 2004.

Sabo Technical Center: Organizing the Quantity of Landslide Movement, 2001.

Tsunaki, R., Fujisawa, K., Matayoshi, H., and Yanagihara, K.: 1998 Survey of Landslide Risk Locations, Internal Documents of the Landslide Division, Erosion Control Department, of the Public Works Research Institute, Ministry of Construction, 31-37, 1999.

Japan Society of Civil Engineers, Committee on Rock Mechanics, Subcommittee on Slope Stability: Rock Slope Surveying and Countermeasures, 1999.

Association of Precise Survey and Applied Technology: Progress of Surveying Technology, Fifteenth Anniversary Project Documents, 1995.

Japan Association for Slope Disaster Management: Landslide Observation Handbook, 1997.

Ruege, J. M.: Electric Distance Measurement, 3rd Edition, Springer-Verlag, 1990. 